\title{
Nano-Sized Silver Colloids Produced and Stabilized by Amino-Functionalized Polymers: Polymer Structure-Nanoparticle Features and Polymer Structure- Growth Kinetics Relationships
}

\author{
Carin C. S. Batista, ${ }^{a}$ Lindomar J. C. Albuquerque, ${ }^{a}$ Caroline A. S. Ribeiro, ${ }^{a}$ \\ Carlos E. de Castro, ${ }^{a}$ Erica G. A. Miranda, ${ }^{a}$ Iseli L. Nantes, ${ }^{a}$ Brunno L. Albuquerque, ${ }^{b}$ \\ Mateus B. Cardoso ${ }^{c}$ and Fernando C. Giacomelli*,a \\ ${ }^{a}$ Centro de Ciências Naturais e Humanas, Universidade Federal do ABC, \\ 09210-580 Santo André-SP, Brazil \\ ${ }^{b}$ Departamento de Química, Universidade Federal de Santa Catarina, \\ 88040-900 Florianópolis-SC, Brazil \\ ${ }^{c}$ Laboratório Nacional de Luz Sincrotron e Laboratório Nacional de Nanotecnologia, \\ CP 6192, 13083-970 Campinas-SP Brazil
}

\begin{abstract}
The synthesis of highly stable silver colloids has been successfully achieved by using reducing/ stabilizer amino-functionalized polymers without the aid of any other external agent. The polymer coated inorganic nanoparticles have been detailed characterized via scattering, imaging and UV-Vis spectroscopy. The combination of transmission electron microscopy (TEM) and light scattering suggested nearly spherical structures stabilized by a lengthy polymeric shell with surface charge dependent on polymer nature and molecular weight. The hybrid nanomaterials are produced via an autocatalytic process where the onset of colloid formation is influenced by the available hydrophobic nitrogen units normalized by the weight of polymer. The induction period, however, does not influence the dimension of the metallic cores which are similar although with a high degree of dispersity. The coating confers a large hydrodynamic size to the silver nanoparticles and the length of the stabilizing shell is chiefly governed by the molecular weight of the aminofunctionalized polymers. These features may significantly impact the cytotoxicity and bactericidal properties of the hybrid materials. These investigations are currently underway.
\end{abstract}

Keywords: silver nanoparticles, amino-functionalized polymers, autocatalysis, structure, kinetics

\section{Introduction}

Silver nanoparticles (AgNPs) have proven to be very useful in applications ranging from catalysis, ${ }^{1}$ environmental treatments, ${ }^{2}$ medical devices ${ }^{3}$ and many others. Particularly considering the field of biomedical nanotechnology, these engineered noble metal particles find clinical applications in antimicrobial treatments, ${ }^{4}$ drug delivery, detection and imaging. ${ }^{5}$ This is essentially due to their unique optical and outstanding bactericidal properties. ${ }^{6,7}$ The antibacterial behavior of AgNPs comes most probably from adhesion and accumulation of AgNPs onto the bacterial surface thereby damaging cell membranes and enhancing permeability. ${ }^{8}$ Additionally, the

*e-mail: fernando.giacomelli@ufabc.edu.br elution of silver ions which can be easily uptaken by the cells and further bind to negatively charged compounds can contribute to such property. ${ }^{9,10}$ The biological activity of AgNPs is sensitive to size, shape, concentration, surface charge and chemical nature of stabilizers. ${ }^{11}$ These physicochemical parameters are primarily dependent on the strategies to produce the colloidal suspensions.

Hence, and following early developments in wet chemistry methods such as the well-known reduction of silver nitrate $\left(\mathrm{AgNO}_{3}\right)$ by sodium borohydride $\left(\mathrm{NaBH}_{4}\right)$ or sodium citrate $\left(\mathrm{Na}_{3} \mathrm{C}_{6} \mathrm{H}_{5} \mathrm{O}_{7}\right)$ in the presence of low molecular weight stabilizers, ${ }^{12}$ the use of polymers and copolymers is clearly an interesting route for the fabrication of polymer-stabilized metallic nanoparticles. ${ }^{13-15}$ This approach enables a single step synthesis without using external agents since the polymer incorporation into 
metallic nanoparticles manufacturing can simultaneously play the roles of reductant and stabilizer (capping) if appropriated functional groups are present. This strategy has been recently applied by us in the synthesis of AuNPs at the presence of $\mathrm{pH}$-responsive amino-functionalized amphiphilic block copolymers. ${ }^{16}$ The reduction of $\mathrm{Au}^{\mathrm{III}}$ species was induced by the presence of the lone pair of electrons in the nitrogen atoms of the amino groups. The polymer chains subsequently chemisorb onto the newly generated particle promoting colloidal stability.

Herein, we address this issue in relation to the synthesis and stabilization of nano-sized silver colloids by using the amino-functionalized polymers PEI (polyethylenimine), PVP (polyvinylpyrrolidone) and PEO- $b$-P2VP (poly(ethylene oxide)- $b$-poly(2-vinyl pyridine)). Their reduction capability is supposed to be also linked to the presence of the hydrophobic nitrogen atoms and they further promote electrostatic and/or steric stabilization to the metallic colloids. Hitherto, we demonstrate the polymer structure-growth kinetics and polymer structurenanoparticle features relationships. The structural and kinetics investigations have been performed by using UV-Vis spectroscopy, static and dynamic light scattering (SDLS), electrophoretic light scattering (ELS), smallangle X-ray scattering (SAXS) and imaging (transmission electron microscopy, TEM). We consider important to highlight that further investigations are currently underway in order to correlate the polymer nature and molecular weight to cytotoxicity and bactericidal properties of the hybrid nanomaterials.

\section{Experimental}

\section{Materials}

Silver nitrate $\left(\mathrm{AgNO}_{3}\right)$ and ethylene glycol (EG) were of analytical grade purchased from Sigma-Aldrich and used as received. The polymers PEI (polyethyleneimine) and PVP (polyvinylpyrrolidone) were also purchased from Sigma-Aldrich whereas PEO- $b$-P2VP block copolymer (poly(ethylene oxide)- $b$-poly(2-vinyl pyridine)) was purchased from Polymer Source Inc. The water was pretreated with Milli-Q ${ }^{\circledR}$ Plus System (Millipore Corporation). The Figure 1 portrays the molecular structure of the reducing/stabilizer polymers and their respective molecular characteristics are given in Table 1.

\section{Nanoparticle synthesis}

The synthesis of the AgNPs was carried out based on the procedures described by Silvert et al. ${ }^{17}$ The silver
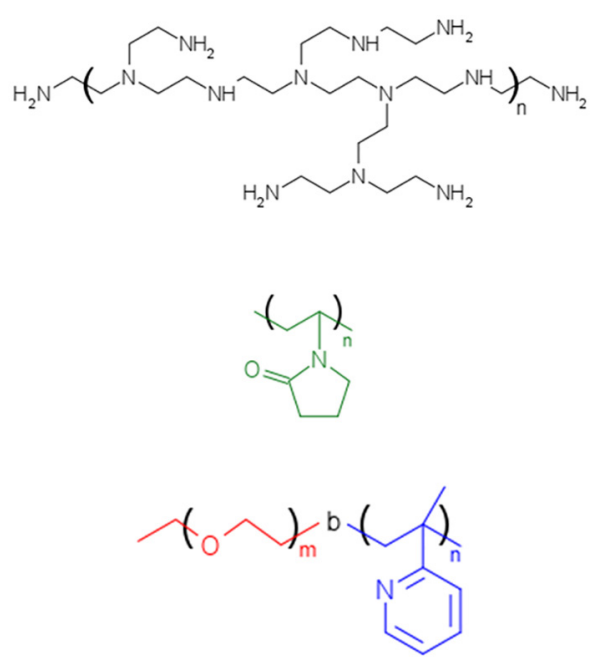

Figure 1. Molecular structure of the reducing/stabilizer polymers: branched polyethyleneimine (top), polyvinylpyrrolidone (middle) and poly(ethylene oxide)- $b$-poly(2-vinyl pyridine) (bottom).

Table 1. Molecular characteristics of the reducer/stabilizer polymers

\begin{tabular}{lcc}
\hline Polymer $^{a}$ & $M_{n} /\left(\mathrm{g} \mathrm{mol}^{-1}\right)$ & $M_{w} / M_{n}$ \\
\hline BLPEI & 1200 & 1.1 \\
BHPEI & 25000 & 2.5 \\
LPVP & 40000 & 2.0 \\
HPVP & 58000 & 2.3 \\
PEO- $b$-P2VP & $21000-b-13500$ & 1.1 \\
\hline
\end{tabular}

${ }^{\mathrm{a}} \mathrm{B}, \mathrm{L}$ and $\mathrm{H}$ stand, respectively, for branched, lower molecular weight and higher molecular weight.

colloids were produced in EG environment containing molecularly dissolved PEI, PVP or PEO- $b$-P2VP polymer chains. The $\mathrm{AgNO}_{3}$ /polymer weight ratio was an initial variable of investigation. The silver nitrate $(0.011 \mathrm{mg})$ was slowly added to the solution containing EG and the reducing/stabilizer polymers. The system was then homogenized, heated up to $50{ }^{\circ} \mathrm{C}$ and the reactions were carried out for 2 hours. Whenever noticed, the manufactured AgNPs were precipitated in acetone followed by centrifugation $\left(4{ }^{\circ} \mathrm{C}, 11000 \mathrm{rpm}, 30 \mathrm{~min}\right)$ and resuspension in water.

\section{Techniques of characterization}

\section{Dynamic light scattering (DLS)}

DLS measurements were performed using an ALV/CGS-3 compact goniometer system consisting of a $22 \mathrm{~mW}$ HeNe linearly polarized laser operating at a wavelength of $633 \mathrm{~nm}$, an ALV 7004 digital correlator and a pair of avalanche photodiodes operating in pseudo cross-correlation mode. The samples were placed in 
$10 \mathrm{~mm}$ diameter glass cells and maintained at a constant temperature of $25 \pm 1{ }^{\circ} \mathrm{C}$. The autocorrelation functions reported are based on 3 independent runs of $60 \mathrm{~s}$ counting time. The data were collected and further averaged by using the ALV Correlator Control software. The correlation functions were analyzed using the nonlinear inverse Laplace transformation algorithm CONTIN resulting in distributions of relaxation times $A(\tau)$. The hydrodynamic radius $\left(R_{\mathrm{H}}\right)$ of the nanoparticles was determined by using the Stokes-Einstein relation with $D=\tau^{-1} q^{-2}$ :

$R_{H}=\frac{k_{B} T q^{2}}{6 \pi \eta} \tau$

where $k_{B}$ is the Boltzmann constant, $T$ is the absolute temperature, $q$ is the scattering vector, $\eta$ is the viscosity of the solvent and $\tau$ is the mean relaxation time related to the diffusion of the nanoparticles. The distributions of relaxation times were also converted to distributions of $R_{\mathrm{H}}$ by using the Stokes-Einstein equation. The polydispersity of the nanoparticles was accessed by using the cumulant analysis ${ }^{18}$ of the autocorrelation functions measured at $90^{\circ}$ as:

$\ln g_{1}(t)=\ln C-\Gamma t+\frac{\mu_{2}}{2} t^{2}$

where $C$ is the amplitude of the correlation function and $\Gamma$ is the relaxation frequency $\left(\tau^{-1}\right)$. The parameter $\mu_{2}$ is known as the second-order cumulant and it was used to compute the polydispersity index of the samples (PDI $=\mu_{2} / \Gamma^{2}$ ).

\section{Static light scattering (SLS)}

The SLS measurements were carried out by varying the scattering angle $(\theta)$ from 30 to $150^{\circ}$ with a $5^{\circ}$ stepwise increase. At each angle, the light scattering intensity was measured in triplicate and the averaged values are reported. The radius of gyration $\left(R_{\mathrm{G}}\right)$ of the structures was estimated using the Guinier approach:

$\ln I_{s c}(q)=\ln I_{0}-\frac{R_{G}^{2}}{3} q^{2}$

hence, measuring the light scattering intensity $\left(I_{\mathrm{sc}}\right)$ at a given angular range for one single diluted concentration, the value of $R_{\mathrm{G}}$ can be estimated from the slope $\left(I_{0}\right.$ is the forward scattering).

\section{Electrophoretic light scattering (ELS)}

ELS measurements were used to determine the average zeta potential $(\zeta)$ of the polymer-stabilized silver colloids.
The values were collected using a Zetasizer Nano-ZS ZEN3600 instrument (Malvern Instruments, UK). This instrument measures the electrophoretic mobility $\left(U_{\mathrm{E}}\right)$ and converts the value to $\zeta$-potential $(\mathrm{mV})$ through Henry's equation:

$U_{E}=\frac{2 \varepsilon \zeta f(k a)}{3 \eta}$

where $\varepsilon$ is the dielectric constant of the medium and $\eta$ its viscosity. Furthermore, $f(k a)$ is the Henry's function, which was calculated through the Smoluchowski approximation $f(k a)=1.5$. Each $\zeta$-potential value reported in the manuscript is an average of 10 independent measurements with repeatability $\pm 2 \%$.

\section{Small angle X-ray scattering (SAXS)}

Small angle X-ray scattering (SAXS) measurements were performed at the SAXS1 beamline of the Brazilian Synchrotron Light Laboratory (LNLS, Campinas-SP, Brazil). The sample holder consisted of a temperaturecontrolled vacuum flow-through cell composed of two mica windows. ${ }^{19}$ The collimated beam crossed the samples through an evacuated flight tube and was scattered to a Pilatus 300K 2D detector (Dectris). In order to cover the desired $q$ range (from 0.06 to $1.20 \mathrm{~nm}^{-1}$ ) where $\mathrm{n}$ ca. 1 for X-rays, the sample-to-detector distance was set to $1998.3 \mathrm{~mm}$ (silver behenate was used for sample-to-detector distance calibration). The data were collected after an exposure time of $100 \mathrm{~s}$. The 2D scattering patterns were found to be isotropic and they were normalized by the sample transmission undertaken using the FIT2D software. The $I(q) v s . q$ scattering curves were corrected by subtracting the pure solvent scattering.

\section{Transmission electron microscopy (TEM)}

The TEM samples were prepared by evaporation of 5 drops $(4 \mu \mathrm{L})$ of the colloidal samples into copper grids (400 square mesh) coated with formvar. The micrographs were acquired with a JEOL JEM 1011 electron microscope operating at $100 \mathrm{kV}$. The size histograms were made by measuring at least 130 particles using ImageJ and adjusted with a Gaussian distribution equation to obtain the average sizes and deviation.

\section{UV-Vis spectroscopy}

UV-Vis spectroscopy data were acquired by using a Varian Cary 50 spectrometer. The spectral resolution for wavelength scanning was $1.0 \mathrm{~nm}$. The measurements were performed by using a quartz cell with optical path length of $1.0 \mathrm{~cm}$. 


\section{Results and Discussion}

\section{Polymer structure-nanoparticle features relationships}

The AgNPs were initially produced by changing the silver nitrate/polymer weight ratio $\left(\mathrm{AgNO}_{3} /\right.$ polymer $)$ as reported in Table 2. The data evidences that larger nanoparticles were obtained when higher amounts of polymer were used. This trend is possibly related to higher amount of polymer taking part of the assemblies. Furthermore, the use of small concentrations of the reducing/stabilizer polymers $\left(\mathrm{AgNO}_{3} /\right.$ polymer $\left.>6.8\right)$ led to the formation of micrometer-sized aggregates and/or solid precipitates visible even by the naked eye. On the other hand, the use of high polymer amounts $\left(\mathrm{AgNO}_{3} /\right.$ polymer < 6.8) does not improve the stability of the produced colloids. Therefore, we consider $\mathrm{AgNO}_{3} /$ polymer $=6.8$ the most appropriated ratio at the investigated range to produce stable silver nanoparticles. Accordingly, the whole set of remaining data (unless otherwise noticed) has been conducted by keeping fixed this parameter. In such condition, the AgNPs remained stable for months when stored at $4{ }^{\circ} \mathrm{C}$.

Table 2. Light scattering data determined for AgNPs produced and stabilized by using different amino-functionalized polymers at different $\mathrm{AgNO}_{3} /$ polymer weight ratios

\begin{tabular}{lccccc}
\hline Polymer & Weight ratio & $R_{\mathrm{H}} / \mathrm{nm}$ & $\mathrm{PDI}$ & $R_{\mathrm{G}} / \mathrm{nm}$ & $\rho=R_{\mathrm{G}} / R_{\mathrm{H}}$ \\
\hline \multirow{2}{*}{ BLPEI } & 3.4 & 31.6 & 0.18 & - & - \\
& 6.8 & 30.6 & 0.24 & 33.8 & 1.1 \\
& 34.2 & 23.0 & 0.12 & - & - \\
\hline \multirow{2}{*}{ BHPEI } & 3.4 & 53.3 & 0.16 & - & - \\
& 6.8 & 37.4 & 0.12 & 28.2 & 0.8 \\
& 34.2 & 54.5 & 0.22 & - & - \\
\hline \multirow{2}{*}{ LPVP } & 3.4 & 50.2 & 0.19 & - & - \\
& 6.8 & 40.3 & 0.16 & 50.9 & 1.2 \\
& 34.2 & 43.1 & 0.18 & - & - \\
\hline HPVP & 3.4 & 76.8 & 0.23 & - & - \\
& 6.8 & 42.3 & 0.20 & 51.6 & 1.2 \\
& 34.2 & 35.6 & 0.19 & - & - \\
\hline & 3.4 & 84.3 & 0.18 & - & - \\
& 6.8 & 56.1 & 0.16 & 56.0 & 1.0 \\
& 34.2 & 52.1 & 0.23 & - & - \\
\hline
\end{tabular}

The Figure 2 portrays the UV-Vis spectra (top) and the visual appearance of the produced AgNPs (bottom) by using different reducing/stabilizer polymers. The observed absorption at ca. $420 \mathrm{~nm}$ is due to the characteristic localized surface plasmon resonance of AgNPs as the result of the collective oscillation of free surface electrons. This is indeed the fingerprint on the formation of the silver colloids along to their pale yellow color. The $\lambda_{\max }$ is roughly the same comparing HPVP, LPVP and BHPEI whereas for BLPEI and $\mathrm{PEO}-b$-P2VP, respectively, a blue and a red-shift were monitored. It is well established that $\lambda_{\max }$ and bandwidth are dependent on particle size, dielectric medium and surfaceadsorbed species. ${ }^{20}$ Accordingly, since the stabilizers are different in nature and size, the evidences cannot be simply attributed to one effect alone or by a combination of effects. Nevertheless, $\lambda_{\max }$ ca. $420 \mathrm{~nm}$ is typical of spherical AgNPs with some degree of size dispersity. ${ }^{21}$ Additionally, the UVVis spectrum in the presence of PEO- $b$-P2VP is slightly different from the others. This can be related to a different adopted conformation of the soft material surrounding the metallic core as discussed hereafter.
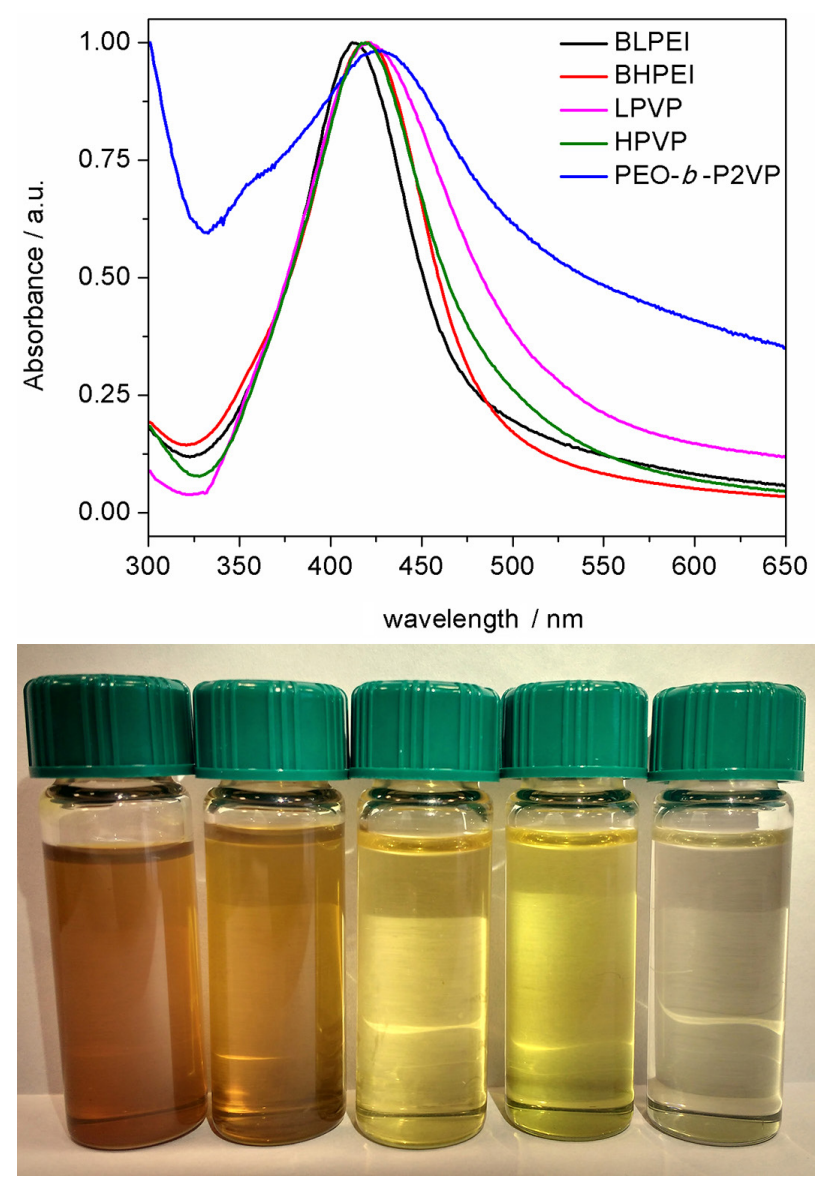

Figure 2. UV-Vis spectra of AgNPs produced in EG at weight ratio 6.8 by using different amino-functionalized polymers according to the legend (top); visual appearance of the produced AgNPs (bottom) after 2 hours of reaction, from left to right: BLPEI, BHPEI, LPVP, HPVP, PEO- $b$-P2VP.

The structure of the hybrid nanoclusters has been subsequently characterized via scattering and imaging. Representatively, the Figure 3A portrays the autocorrelation functions for AgNPs produced in EG and stabilized by 
using HPVP as a function of the scattering angle. The single relaxation mode $(\Gamma=1 / \tau)$ was plotted as a function of $q^{2}$ and representative examples for AgNPs produced and stabilized by BLPEI, HPVP and PEO- $b$-P2VP are given in Figure 3B. The linear $\Gamma$ vs. $q^{2}$ behavior is representative of a diffusive movement which accordingly means that mean hydrodynamic radii $\left(R_{\mathrm{H}}\right)$ can be calculated from the diffusion coefficients $\left(D=\Gamma \times q^{-2}\right)$ using the straightforward Stokes-Einstein relation. Qualitatively, one can notice different slopes by looking at the $\Gamma v s . q^{2}$ profiles (the higher the slope, the smaller the $R_{\mathrm{H}}$ of the AgNPs).
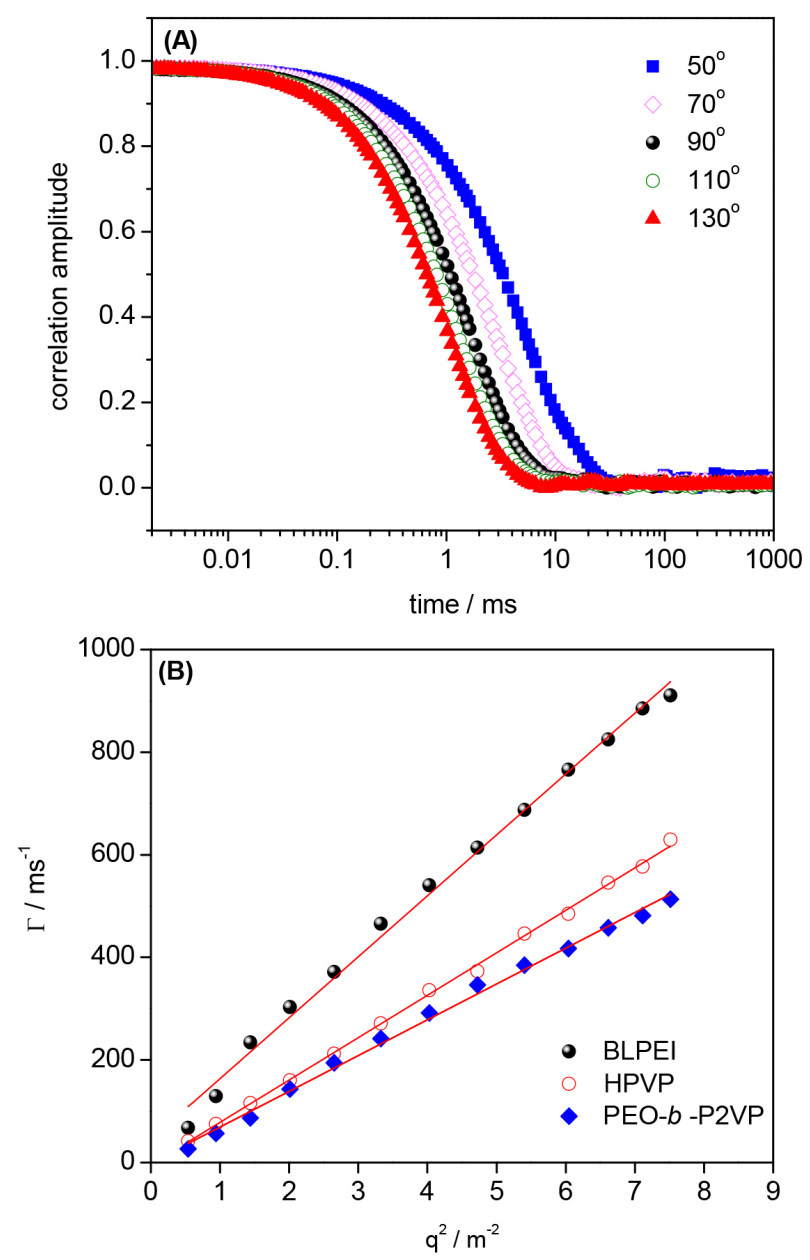

Figure 3. Autocorrelation functions measured as a function of the scattering angle for AgNPs produced in EG and stabilized by HPVP (A); relaxation frequency $(\Gamma=1 / \tau)$ as a function of $q^{2}$ for AgNPs produced and stabilized by BLPEI, HPVP and PEO- $b$-P2VP according to the legend (B).

The autocorrelation functions measured at $90^{\circ}$ were firstly analyzed by using the nonlinear inverse Laplace transformation algorithm CONTIN resulting in distributions of sizes (Figure 4A). The presence of one single distribution of size regardless the condition allowed determining nanoparticle size via the cumulant analysis which consequently permitted also the determination of polydispersity indexes. Moreover, the radius of gyration $\left(R_{\mathrm{G}}\right)$ was estimated by means of static light scattering using the Guinier approach and the representative data for BLPEI, HPVP and PEO- $b$-P2VP are given in Figure 4B. The complete set of numbers acquired by light scattering is given in Table 2 .
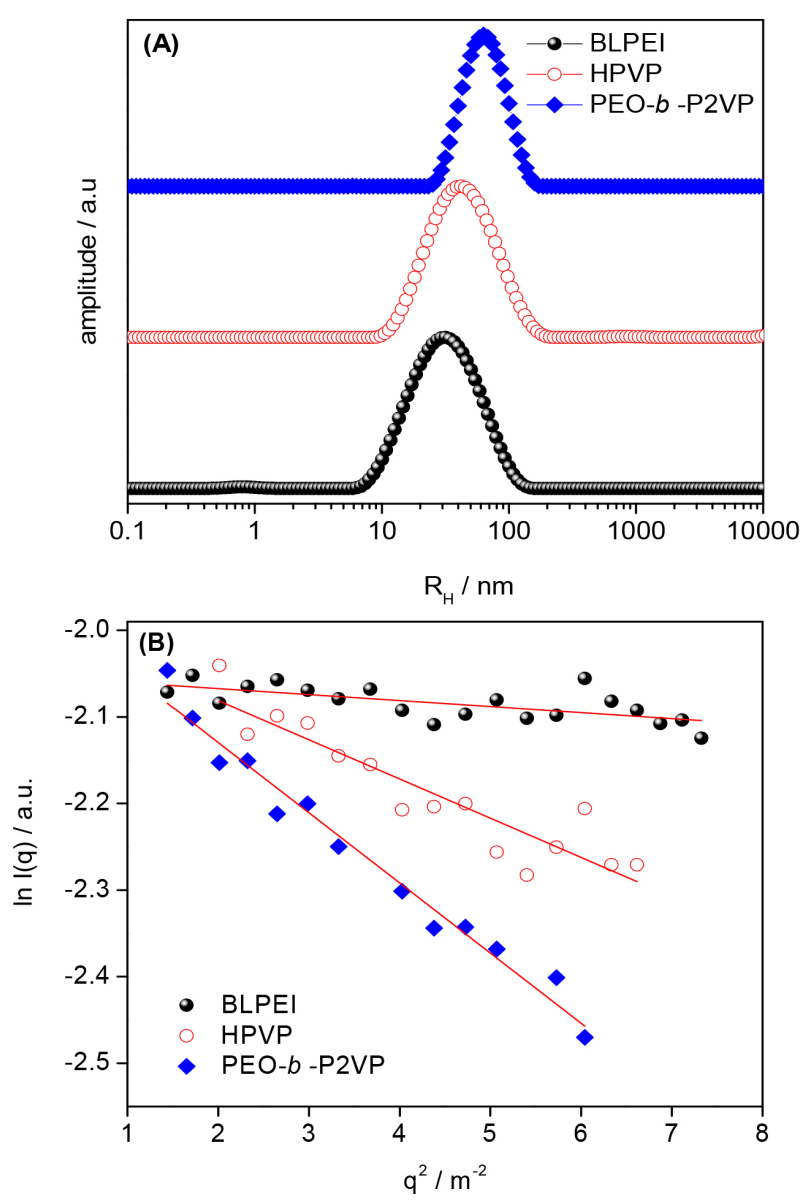

Figure 4. Distribution of sizes obtained from the ACF's measured at $90^{\circ}$ (A); Guinier plots (B) for AgNPs produced by using reducing/stabilizer polymers according to the legend.

The scattering characterization of the AgNPs has been complemented by TEM imaging. The micrographs portrayed in Figure 5 evidences that all samples are indeed polydisperse. The images convincingly show the formation of nearly spherical or spherical objects. This is also supported by the combination of static and dynamic light scattering data which provides information on the shape and inner structure of scattering objects. It is well established that the ratio $\rho=R_{\mathrm{G}} / R_{\mathrm{H}}$ is a characteristic parameter related to the shape or conformation of scatters. For hard-spheres, coils and rod-like structures $\rho$-values of $0.775,1.78$ and $\geq 2$ have been respectively reported..$^{22}$ Furthermore, the $\rho$-value of spherical objects is dependent on their inner structure and compactness ${ }^{23}$ being close to 

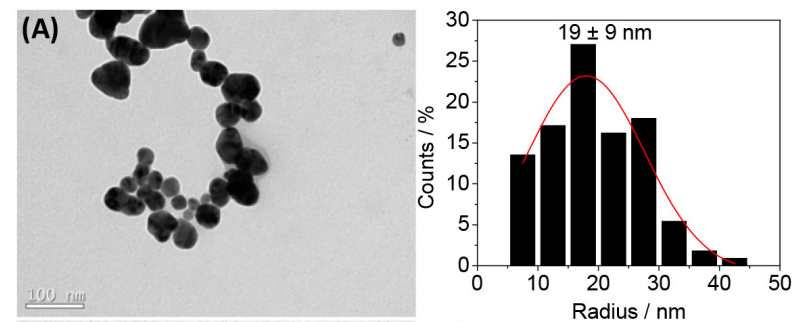

(B)
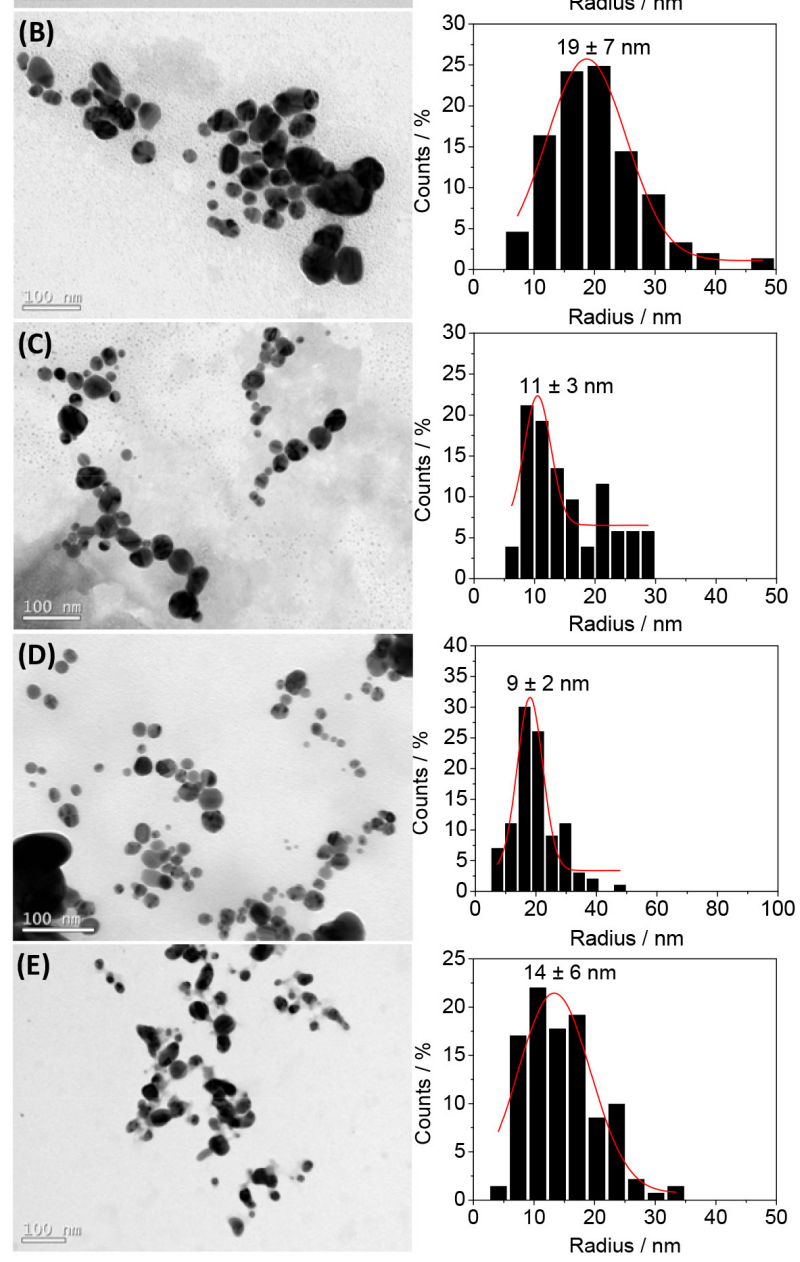

Figure 5. TEM micrographs along to the respective size distribution histograms for AgNPs produced and stabilized by using aminofunctionalized polymers: (A) BLPEI, (B) BHPEI, (C) LPVP, (D) HPVP, (E) PEO- $b$-P2VP.

0.775 for compact spheres and $\rho$ ca. $0.8-0.9$ for block copolymer micelles due to solvation phenomena. ${ }^{24}$ Indeed, $R_{\mathrm{G}} / R_{\mathrm{H}}$ ratio gives informations on draining properties and density distribution inside spherical particles. The $R_{\mathrm{G}} / R_{\mathrm{H}}$ ratio of highly swollen structures such as random polycondensates (so-called soft balls) and star molecules is about 1.0. In the current investigations, the dimensionless structure-sensitive parameter $\left(R_{\mathrm{G}} / R_{\mathrm{H}}\right)$ was determined to be in the range 0.8-1.2 (Table 2). This suggests that the hard metallic silver cores are surrounded by lengthy stabilizing shells which are certainly highly swollen by water due to their hydrophilic nature.
Additionally, the mean sizes determined from TEM analysis were substantially smaller than those obtained by dynamic light scattering. This discrepancy is, in part, due to dehydration caused by solvent evaporation during casting. However, discrepancies are also expected because DLS reports an intensity-average diameter whilst TEM reports a number-average diameter. Therefore, for a given size distribution of finite polydispersity, TEM images usually undersize relative to DLS. Most important, the TEM measurements allow only the visualization of the metallic core of the assemblies since the electron density of silver overwhelms that of the polymeric shell. The radii of the AgNPs as determined from TEM images are roughly in the range $10-20 \mathrm{~nm}$. Hence, the combination of TEM and DLS suggests that the nanomaterials are composed by a small metallic core surrounded by a lengthy polymeric shell. This feature may have important impacts on cytotoxicity and/or antibacterial properties and will be addressed in a forthcoming paper.

The reported DLS sizes essentially reflect the molecular weight of the stabilizers and/or their conformation. The size increases in the order BLPEI $<$ BHPEI $<$ LPVP $<$ HPVP $<$ PEO- $b$-P2VP. On the other hand, taking into account the TEM size histograms and the high polydispersity evidenced particularly for PEI-stabilized AgNPs $(19 \pm 9$ and $19 \pm 7 \mathrm{~nm}$ for BLPEI and BHPEI, respectively), the radius of the metallic silver cores is not substantially different for the whole set of produced particles. The light scattering results presented in Table 2 imply then that the smaller sizes of the PEI-stabilized AgNPs may be attributed to a thinner stabilizing shell as a consequence of the lower molecular weights of BLPEI and BHPEI compared for instance to PVP polymers. Considering the molecular weight of PEO- $b$ P2VP, it was expected a smaller size of PEO- $b$-P2VP-coated AgNPs as compared to the PVP-coated counterparts. This is truly not the case. Therefore, the higher size of PEO- $b$ P2VP-coated AgNPs is likely influenced by a different conformation of the block copolymer surrounding the silver core. The high degree of hydrophobicity of the P2VP block ${ }^{25}$ may favor $\mathrm{Ag}^{0}-\mathrm{P} 2 \mathrm{VP}$ intermolecular interactions thereby conducting to adsorption of the polymer onto the metallic surface. The packed conformation of the P2VP block thus stabilized by a PEO shell seems to be the more realistic scenario taking into account respectively the hydrophobic and hydrophilic features of P2VP and PEO (Figure 6, right). Such distinct arrangement may be also the reason of the dissimilar UV-Vis absorption profile (Figure 2).

Additionally, the TEM images portray the presence of much smaller particles besides the population of the larger. The presence of smaller particles was indeed not detected by light scattering since the technique is very much 

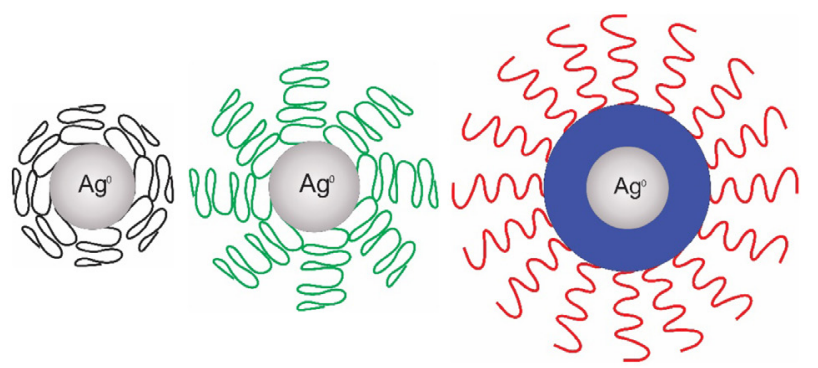

Figure 6. Hypothesized representation of polymer coated AgNPs: PEI (left); PVP (middle); PEO- $b$-P2VP (right).

sensitive to the molar mass and/or size of the scatters. This nevertheless implies a high degree of size dispersity which was also confirmed by SAXS measurements (representative examples for BHPEI and HPVP stabilized AgNPs are shown in Figure 7). The SAXS profiles could be fitted by using the form factor of homogeneous spheres as:

$$
\begin{aligned}
I(q)= & V_{p}^{2} \Delta \sigma^{2} P(q, R)= \\
& \left(\frac{4}{3} \pi R^{3} \Delta \sigma\right)^{2}\left(\frac{3[\sin (q R)-q R \cos (q R)]}{(q R)^{3}}\right)^{2}
\end{aligned}
$$

The sample dispersity was taking into account by using the log-normal distribution that has the probability density function given by:

$f(R, \mu, \sigma)=\frac{1}{\sqrt{2 \pi} \sigma R} \exp -\frac{\ln (R / \mu)^{2}}{2 \sigma^{2}}$

being $R$ the average radius and the parameters $\mu$ and $\sigma$ are the mean and standard deviation of the distribution. The parameter $\sigma$ gives quantitative information about the particle's dispersity.

Although the fittings approaches described the experimental results reasonably well, they suggest large values of size dispersity $(R=13.3 \mathrm{~nm}$ and $\sigma=0.39$, BHPEI; $R=15.0 \mathrm{~nm}$ and $\sigma=0.48$, HPVP). The respective particle size distributions (Figure 7C) also confirm the wide dispersion therefore in agreement with the TEM analysis. These representative results confirm that the samples have a considerable degree of size dispersity regardless the stabilizer. Nevertheless, the quality of the fittings, particularly at the low- $q$ range, evidences the electrostatic and/or steric stabilization provided to the AgNPs by the polymer chains since it means the absence of large ill-aggregates. Finally, the excess scattering at the tail of the SAXS curves (high- $q$ range) is presumably related to free polymer chains still present at the EG environment.

The structural characterization was concluded by electrophoretic light scattering measurements. The
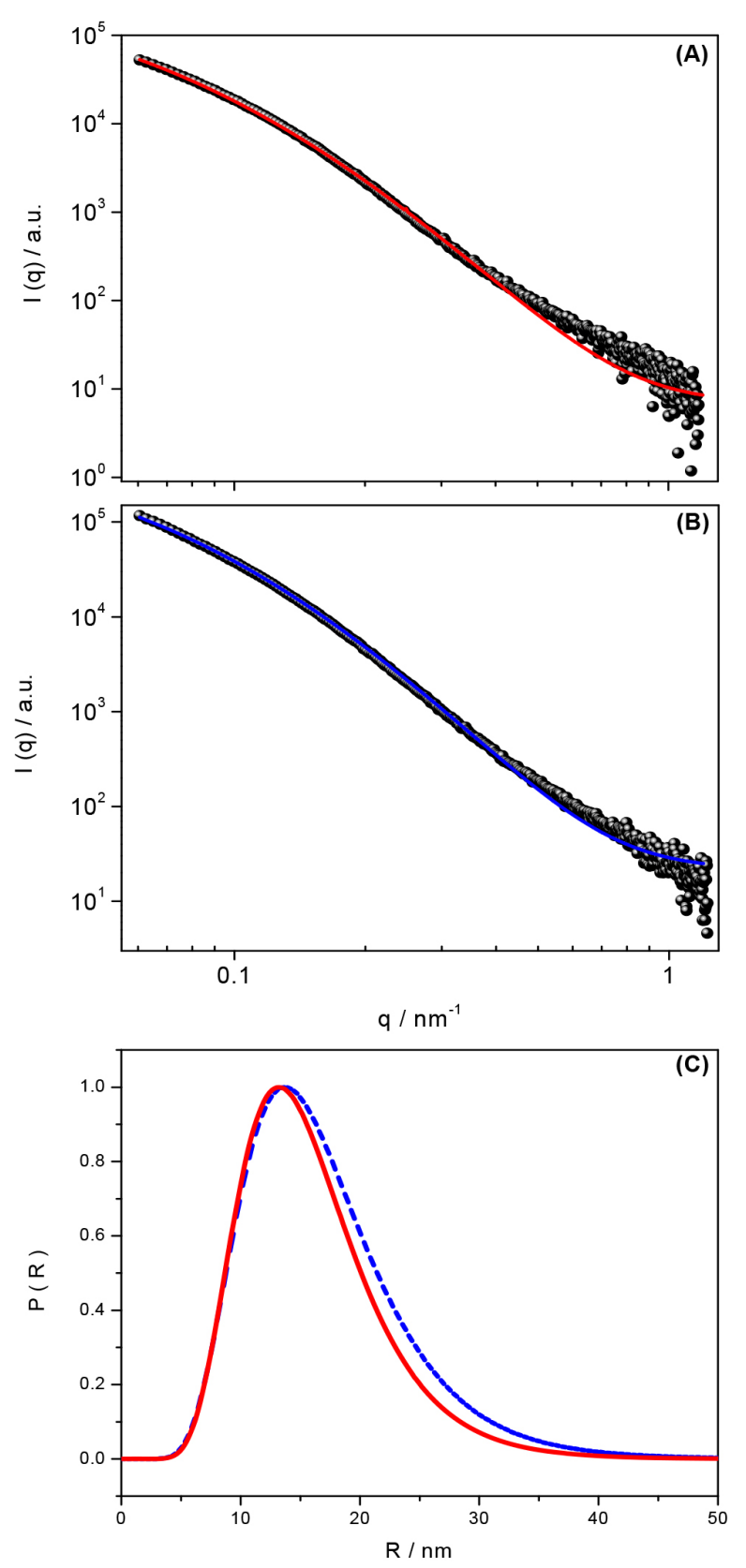

Figure 7. SAXS profiles (circles) and corresponding curve fittings (lines) for AgNPs produced and stabilized by BHPEI (A) and HPVP (B). Respective particle size distributions (C) for BHPEI (solid line) and HPVP (dotted line).

Figure 8 portrays the average $\zeta$-potential as a function of the stabilizer. These experiments were conducted after resuspending the assemblies in water.

Clearly, the surface charge of the stabilized silver colloids is polymer-dependent. The surface of the nanoparticles is positively charged whenever stabilized by PEI and negatively charged when the reducing/stabilizer agent is PVP. The surface charge is roughly neutral for the case of PEO- $b$-P2VP. The measured $\mathrm{pH}$ of the solutions containing PVP-stabilized AgNPs was $\mathrm{pH}$ ca. 6. The pKa of 


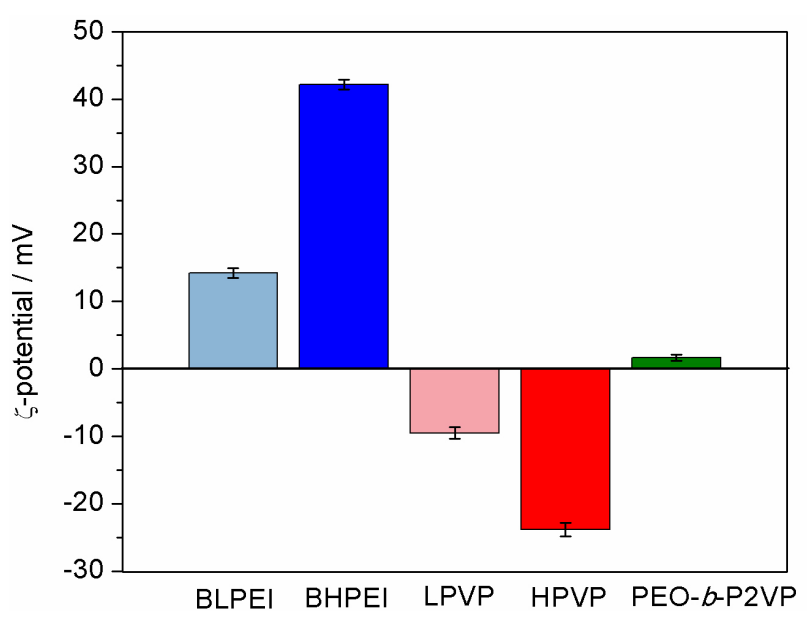

Figure 8. Average $\zeta$-potential of AgNPs stabilized by different aminofunctionalized polymers according to the $\mathrm{x}$-axis.

PVP units is at about pKa ca. $5^{26}$ which accordingly means that the polymer is essentially neutral in such condition. The negative $\zeta$-potential promoted by a neutral polymer is interesting and results in both electrostatic and steric stabilization of the nanoparticles. The steric stabilization is mainly due to repulsion forces generated by the PVP steric configurations whereas the negative $\zeta$-potential is probably the consequence of charge partitioning on carbonyl groups present in the PVP polymer backbone. Similar behavior is usually observed in non-ionic polyesterbased nanoparticles. ${ }^{27}$ The more negative $\zeta$-potential of the HPVP-stabilized AgNPs implies certainly higher polymer amounts stabilizing the silver colloids. This is presumably linked to the higher molecular weight of HPVP. On the other hand, the PEO- $b$-P2VP provides a nearly neutral $\zeta$-potential to the assemblies meaning a shielded surface. The pKa of P2VP units is also at about pKa ca. 5. ${ }^{28,29}$ Therefore, the outer shell does not exhibit ionizable groups and there is no charge partitioning which could possibly lead to a negative or positive surface. Although the AgNPs exhibit a nearly neutral surface, the nanoparticles were found to be remarkably stable for months, thanks probably to the steric stabilization promoted by the PEO segment. Similar interpretation was suggested previously for non-charged carbohydrate-coated nanoparticles. ${ }^{30,31}$

The positively charged PEI-stabilized AgNPs is more clearly understandable if one considers the measured solution $\mathrm{pH}$ ( $\mathrm{pH}$ ca. 7.2). The branched PEI holds three pKa values (4.5 for primary, 6.7 for secondary and 11.6 for tertiary amine groups). ${ }^{32}$ Accordingly, at such $\mathrm{pH}$ environment the tertiary amines are fully ionized whereas the secondary amines are at least partially ionized conferring residual positive charges to the polymer chains which then electrostatically stabilize the metallic assemblies. As it was evidenced for PVP, the magnitude of the surface charge depends on the molecular weight being higher when BHPEI was used. Since the monitored $\mathrm{pH}$ value of both (BLPEI and BHPEI) suspensions was roughly the same, this can only be attributed again to a higher amount of the catiomer stabilizing the noble metal nanoparticles.

\section{Polymer structure-growth kinetics relationship}

The growth kinetics has been scanned via UV-Vis spectroscopy. Representatively, the Figure 9A portrays the UV-Vis spectra evolution for AgNPs produced in ethylene glycol in presence of BLPEI. The kinetic profiles (absorbance at $\lambda_{\max } v s$. time) for various polymers are depicted in Figure 9B at the same weight ratio.

One sees in Figure 9A that $\lambda_{\max }$ remains essentially at the same position with progressive absorption increase over time. The same behavior has been observed in all measurements although the evolution in time is polymerdependent. The absorption increase as a function of time is therefore the result of the progressive formation of higher amounts of AgNPs with similar characteristics (since it was not observed considerable differences in $\lambda_{\max } v s$. time). The AgNPs growth follows a sigmoidal behavior typically observed in autocatalytic processes ${ }^{33}$ where ionic silver $\left(\mathrm{Ag}^{+}\right)$is reduced on the surface of growing $\mathrm{Ag}^{0}$ clusters. The process starts with an induction period where it takes place the characteristic nucleation step leading to critically-sized aggregates. The fastest onset was observed in the presence of PEI followed by PVP and ultimately PEO- $b$-P2VP. The characteristic onsets of AgNPs formation (induction time) are given in Table 3. The data evidence that the reaction starts significantly later when the block copolymer is used as the $\mathrm{Ag}^{+}$reductant.

The autocatalytic behavior is also confirmed by the linear profiles of $\ln \mathrm{A}_{\mathrm{t}} /\left(\mathrm{A}_{\infty}-\mathrm{A}_{\mathrm{t}}\right) v s$. time. Such behavior is expected for simple autocatalysis. ${ }^{34}$ The slope of the profiles portrayed in Figure 9C refers to the constant $k_{\mathrm{obs}}$ (Table 3) which is the rate constant of $\mathrm{Ag}^{+}$reduction on the surface of $\mathrm{Ag}^{0}$ nucleus. ${ }^{35}$ The fit quality is reasonable $\left(r^{2}>0.92\right)$ and evidence that this reaction step is essentially polymer-independent. The profiles suggest that the particle formation mechanism is essentially the same. Therefore, as soon as the induction time ends after reaching a given concentration of $\mathrm{Ag}^{0}$ nucleus, the autocatalytic surface growth is initiated with similar reaction rates.

Concerning particularly PEO- $b-\mathrm{P} 2 \mathrm{VP}$, based on the kinetic data, it has been performed the same evaluation at $\mathrm{AgNO}_{3} / \mathrm{PEO}-b-\mathrm{P} 2 \mathrm{VP}=1.4$. In such condition, the number of hydrophobic amino groups in the system is nearly the same as present at the condition $\mathrm{AgNO}_{3} / \mathrm{BLPEI}=6.8$. Even by increasing the polymer amount by almost 5 times, it 

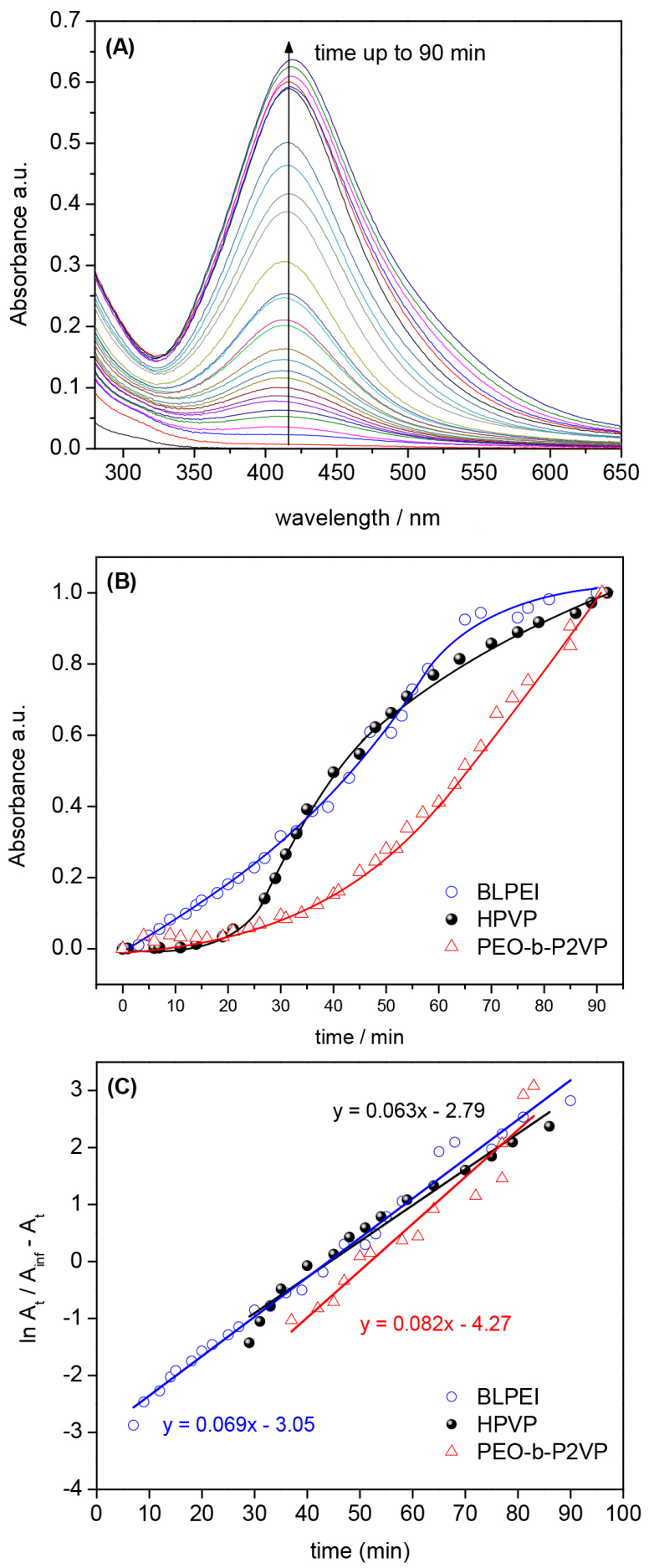

Figure 9. Evolution of UV-Vis spectra during AgNPs synthesis in EG environment containing BLPEI (A); particle growth kinetics: $\lambda_{\max } v s$. time (B) and $\ln \mathrm{A}_{\mathrm{t}} /\left(\mathrm{A}_{\infty}-\mathrm{A}_{\mathrm{t}}\right) v s$. time profiles (C) by using different reducing/ stabilizer polymers according to the legend.

was not observed considerable enhancement in the rate of reaction/induction time (data not shown). It led us to conclude that the behavior cannot be rationalized only by considering the number of available amino groups in the systems. Moreover, since the pKa values for P2VP and
Table 3. Induction time and kinetic constant determined for AgNPs produced by using different amino-functionalized polymers at $\mathrm{AgNO}_{3} /$ polymer weight ratio $=6.8$

\begin{tabular}{lcc}
\hline Polymer & Induction time $/ \mathrm{min}$ & $k_{\mathrm{obs}} / \mathrm{min}^{-1}$ \\
\hline BLPEI & 3 & 0.069 \\
BHPEI & 5 & 0.043 \\
LPVP & 8 & 0.076 \\
HPVP & 25 & 0.063 \\
PEO- $b$-P2VP & 35 & 0.082 \\
\hline
\end{tabular}

PVP are roughly the same (pKa ca. 5), and taking into account the measured $\mathrm{pH}$ ( $\mathrm{pH}$ ca. 6), the differences in the onset of reaction cannot be attributed to different sources of electrons at different amine units. Apparently, the behavior is thus linked to the number of nitrogen atoms per weight of polymer. We herein highlight that the investigations were performed by keeping fixed the polymer weight (rather than the number of amine units) in order to not rule out formerly the other sources of electrons (such as the oxygen atoms) which could also promote $\mathrm{Ag}^{+}$reduction. Indeed, the PEO block may also, at least to some extent, influence the $\mathrm{Ag}^{+}$ reduction since the well-known Pluronics (PEO-b-PPO$b$-PEO block copolymers) were evidenced to efficiently reduce $\mathrm{Au}^{3+}$ thereby promoting the formation of AuNPs. ${ }^{14,36}$ However, the herein reported results evidence that the amine units are the main responsible for $\mathrm{Ag}^{+}$reduction. This is clear when one takes into account the same polymer weight $\left(\mathrm{AgNO}_{3} /\right.$ polymer $\left.=6.8\right)$. In such condition, the source of nitrogen atoms in the PEI systems is 2.5 -fold higher whereas in the case of PEO- $b$-P2VP it is 2.4-fold smaller (due to the presence of the hydrophilic PEO segment) as compared to PVP. It accordingly explains the faster and slower reaction rates, respectively, in PEI and PEO- $b$-P2VP environments. This is true even though at the conditions of synthesis the PEI chains are partially ionized (particularly the tertiary amines). Nevertheless, although the source of nitrogen atoms clearly influences that reaction rates, it did not influence the overall size of the silver metallic core since the values determined by TEM are similar if considered the high degree of dispersity.

As a summary, the DLS data evidence that the silver nanoparticles are stabilized by a lengthy stabilizing shell. This is the actual condition at the liquid phase. The size differs from stabilizer to stabilizer reflecting essentially the molecular weight of the amino-functionalized polymers. The polymer features are also responsible for the determined surface charges. The dimension of the metallic core (measured at the dry state), on the other hand, seems not to be substantially influenced by the nature or molecular weight of the stabilizers. 


\section{Conclusions}

The synthesis of highly stable silver colloids has been successfully achieved by using amino-functionalized polymers as simultaneous reductants and stabilizers. The polymer coated inorganic nanoparticles have been detailed characterized by scattering, imaging and UV-Vis spectroscopy, and the whole set of data convincingly reveal the production of nearly spherical nanoparticles polydisperse in size. The structural features of the polymer-coated silver colloids are dependent on polymer nature and molecular weight. The smallest AgNPs were produced using PEI as reductant/stabilizer followed by PVP and ultimately PEO$b$-P2VP. The behavior is claimed to be essentially dependent on the molecular weight of the stabilizer. The metallic hybrid materials are produced via an autocatalytic process where similarly the polymer features play the role. The smallest PEI-stabilized AgNPs are produced significantly faster and the slowest onset of reaction was monitored in PEO- $b$ $\mathrm{P} 2 \mathrm{VP}$ environment. This behavior is apparently linked to the available number of hydrophobic nitrogen units normalized by the weight of polymer. Besides the size, the surface charge of the produced entities is significantly different where PEI, PVP and PEO- $b$-P2VP-stabilized AgNPs hold respectively positive, negative and nearly neutral surfaces. All these features may significantly impact the cytotoxicity and bactericidal properties of the hybrid materials. These investigations are currently underway.

\section{Acknowledgments}

This work has been sponsored by FAPESP (Grants No. 2014/22983-9 and 2014/22322-2) and CNPq (Grant No. 470608/2012-9). F. C. G. and M. B. C. acknowledge the productivity research fellowships granted by $\mathrm{CNPq}$ (Grants No. 302467/2014-9 and 309107/2014-8) and C. E. C. thanks the fellowship granted by FAPESP (Grant No. 2015/24686-4). The CEM at UFABC is acknowledged for the accessibility to the Malvern light scattering equipment. We acknowledge LNLS for SAXS measurements (Project No. 20150021).

\section{References}

1. Elias, W. C.; Eising, R.; Silva, T. R.; Albuquerque, B. L.; Martendal, E.; Meier, L.; Domingos, J. B.; J. Phys. Chem. C 2014, 118, 12962.

2. Tran, Q. H.; Nguyen, V. Q.; Le, A.-T.; Adv. Nat. Sci.: Nanosci. Nanotechnol. 2013, 4, 33001.

3. Majdalawieh, A.; Kanan, M. C.; El-Kadri, O.; Kanan, S. M.; J. Nanosci. Nanotechnol. 2014, 14, 4757.
4. Zazo, H.; Colino, C. I.; Lanao, J. M.; J. Controlled Release 2016, $224,86$.

5. Arvizo, R. R.; Bhattacharyya, S.; Kudgus, R. A.; Giri, K.; Bhattacharya, R.; Mukherjee, P.; Chem. Soc. Rev. 2012, 41, 2943.

6. Dal Lago, V.; de Oliveira, L. F.; Gonçalves, K. A.; Kobarg, J.; Cardoso, M. B.; J. Mater. Chem. 2011, 21, 12267.

7. Yang, C.-H.; Wang, L.-S.; Chen, S.-Y.; Huang, M.-C.; Li, Y.-H.; Lin, Y.-C.; Chen, P.-F.; Shaw, J.-F.; Huang, K.-S.; Int. J. Pharm. 2016, 510, 493.

8. Rai, M.; Kon, K.; Ingle, A.; Duran, N.; Galdiero, S.; Galdiero, M.; Appl. Microbiol. Biotechnol. 2014, 98, 1951.

9. Choi, O.; Hu, Z.; Environ. Sci. Technol. 2008, 42, 4583.

10. Panáček, A.; Kvítek, L.; Prucek, R.; Kolář, M.; Večeřová, R.; Pizúrová, N.; Sharma, V. K.; Nevěčná, T.; Zbořil, R.; J. Phys. Chem. B 2006, 110, 16248.

11. Franci, G.; Falanga, A.; Galdiero, S.; Palomba, L.; Rai, M.; Morelli, G.; Galdiero, M.; Molecules 2015, 20, 8856.

12. de Oliveira, J. F. A.; Cardoso, M. B.; Langmuir 2014, 30, 4879.

13. Yuan, J.-J.; Schmid, A.; Armes, S. P.; Lewis, A. L.; Langmuir 2006, 22, 11022.

14. Sakai, T.; Alexandridis, P.; Langmuir 2004, 20, 8426.

15. Chen, H.; Wang, Y.; Wang, Y.; Dong, S.; Wang, E.; Polymer (Guildf). 2006, 47, 763.

16. Scaravelli, R. C. B.; Dazzi, R. L.; Giacomelli, F. C.; Machado, G.; Giacomelli, C.; Schmidt, V.; J. Colloid Interface Sci. 2013, 397, 114.

17. Silvert, P.-Y.; Herrera-Urbina, R.; Duvauchelle, N.; Vijayakrishnan, V.; Elhsissen, K. T.; J. Mater. Chem. 1996, 6, 573.

18. Stepánek, P.; Dynamic Light Scattering: The Method and Some Applications; Win, B., ed.; Oxford Science Publications: Oxford, 1993.

19. Cavalcanti, L. P.; Torriani, I. L.; Plivelic, T. S.; Oliveira, C. L. P.; Kellermann, G.; Neuenschwander, R.; Rev. Sci. Instrum. 2004, 75, 4541.

20. Mulvaney, P.; Langmuir 1996, 12, 788.

21. Pal, S.; Tak, Y. K.; Song, J. M.; Appl. Environ. Microbiol. 2007, 73, 1712.

22. Sedlák, M.; Koňák, C.; Macromolecules 2009, 42, 7430.

23. Li, M.; Jiang, M.; Zhu, L.; Wu, C.; Macromolecules 1997, 30, 2201.

24. Giacomelli, F. C.; Riegel, I. C.; Petzhold, C. L.; da Silveira, N. P.; Štěpánek, P.; Langmuir 2009, 25, 731.

25. Martin, T. J.; Procházka, K.; Munk, P.; Webber, S. E.; Macromolecules 1996, 29, 6071.

26. Mahltig, B.; Gohy, J.-F.; Antoun, S.; Jérôme, R.; Stamm, M.; Colloid Polym. Sci. 2002, 280, 495.

27. de Oliveira, A. M.; Jäger, E.; Jäger, A.; Stepánek, P.; Giacomelli, F. C.; Colloids Surf., A 2013, 436, 1092.

28. Gohy, J.-F.; Antoun, S.; Jérôme, R.; Macromolecules 2001, 34 , 7435. 
29. Dupin, D.; Fujii, S.; Armes, S. P.; Reeve, P.; Baxter, S. M.; Langmuir 2006, 22, 3381.

30. de Oliveira, L. F.; de Gonçalves, J. O.; de Gonçalves, K. A.; Kobarg, J.; Cardoso, M. B.; J. Biomed. Nanotechnol. 2013, 9 , 1817.

31. de Barros, H. R.; Cardoso, M. B.; de Oliveira, C. C.; Franco, C. R. C.; Belan, D. L.; Vidotti, M.; Riegel-Vidotti, I. C.; RSC Adv. 2016, 6, 9411.

32. Lee, Y. M.; J. Nanosci. Nanotechnol. 2009, 9, 7467.
33. Dondi, R.; Su, W.; Griffith, G. A.; Clark, G.; Burley, G. A.; Small 2012, 8, 770.

34. Huang, Z. Y.; Mills, G.; Hajek, B.; J. Phys. Chem. 1993, 97, 11542.

35. Harada, M.; Inada, Y.; Nomura, M.; J. Colloid Interface Sci. 2009, 337, 427.

36. Alexandridis, P.; Tsianou, M.; Eur. Polym. J. 2011, 47, 569.

Submitted: September 21, 2016

Published online: November 11, 2016 